\title{
Diagnóstico pré-natal de rabdomioma cardíaco fetal pela ultrassonografia: relato de caso*
}

\author{
Prenatal sonographic diagnosis of fetal cardiac rhabdomyoma: a case report \\ Hélio Antonio Guimarães Filho ${ }^{1}$, Edward Araujo Junior ${ }^{2}$, Cláudio Rodrigues Pires ${ }^{3}$, Lavoisier \\ Linhares Dias da Costa ${ }^{4}$, Luciano Marcondes Machado Nardozza ${ }^{5}$, Rosiane Mattar ${ }^{6}$
}
Resumo Os tumores cardíacos constituem condição rara, com incidência entre 0,17 e 28/10.000 na população ge- ral. Os rabdomiomas são os tumores mais frequentes no período pré-natal. Este trabalho tem como objetivo relatar o caso de um feto com 31 semanas de gestação que apresentava tumoração intracardíaca de gran- des dimensões, com graves repercussões clínicas.
Unitermos: Diagnóstico pré-natal; Ultrassonografia; Massas fetais; Tumores fetais.

Abstract Cardiac tumors constitute a rare condition, affecting $0.17-28 / 10,000$ of the general population. Rhabdomyomas represent the most frequently found type of tumor in the prenatal period. The present report describes the case of a 31-week fetus presenting with a large intracardiac tumor with severe clinical repercussions. Keywords: Prenatal diagnosis; Ultrasonography; Fetal masses; Fetal tumors.

Guimarães Filho HA, Araujo Junior E, Pires CR, Costa LLD, Nardozza LMM, Mattar R. Diagnóstico pré-natal de rabdomioma cardíaco fetal pela ultrassonografia: relato de caso. Radiol Bras. 2009;42(3):203-205.

\section{INTRODUÇÃO}

Exames de diagnóstico por imagem são habitualmente realizados durante a gestação para avaliar afecções relacionadas ao feto $^{(1)}$. Os tumores cardíacos contituem-se em condição rara, com incidência de 0,17 a 28/10.000, segundo estudos de necropsia $^{(2)}$. Recentemente, observou-se incremento no diagnóstico desses tumores durante o período pré-natal, graças à contribuição da ecocardiografia fetal ${ }^{(3,4)}$. A depender da localização intracardíaca do tu-

* Trabalho realizado na Universidade Federal de São Paulo/ Escola Paulista de Medicina (Unifesp/EPM) e no Centro de Treinamento em Ultra-sonografia de São Paulo (Cetrus), São Paulo, SP, Brasil.

1. Doutor, Professor do Centro de Treinamento em Imaginologia (Cetrim), João Pessoa, PB, Brasil.

2. Pós-Doutor, Professor Afiliado do Departamento de Obstetrícia da Universidade Federal de São Paulo/Escola Paulista de Medicina (Unifesp/EPM), São Paulo, SP, Brasil.

3. Doutor, Diretor do Centro de Treinamento em Ultra-sonografia de São Paulo (Cetrus), São Paulo, SP, Brasil.

4. Especialista em Ultrassonografia e Ecocardiografia Fetal, Diretor do Centro de Treinamento em Imaginologia (Cetrim), João Pessoa, PB, Brasil.

5. Doutor, Chefe da Disciplina de Medicina Fetal do Departamento de Obstetrícia da Universidade Federal de São Paulo/Escola Paulista de Medicina (Unifesp/EPM), São Paulo, SP, Brasil.

6. Livre-Docente, Coordenadora do Programa de Pós-graduação do Departamento de Obstetrícia da Universidade Federal de São Paulo/Escola Paulista de Medicina (Unifesp/EPM), São Paulo, SP, Brasil.

Endereço para correspondência: Dr. Hélio Antonio Guimarães Filho. Rua Reinaldo Tavares de Melo, 142/901, Manaíra. João Pessoa, PB, Brasil, 58038-300. E-mail: helioagf@ig.com.br

Recebido para publicação em 8/5/2007. Aceito, após revisão, em 30/7/2007. mor e de suas dimensões, eles podem cursar com arritmias graves, insuficiência cardíaca congestiva, hidropisia, e não raramente, óbito fetal ${ }^{(\mathbf{5})}$. No presente estudo relatamos o caso de um feto no terceiro trimestre da gestação que apresentava volumosa tumoração intracardíaca diagnosticada pela ecocardiografia pré-natal.

\section{RELATO DO CASO}

Paciente com 23 anos de idade, secundigesta, nulípara, gestação na $31^{\mathrm{a}}$ semana, foi encaminhada para estudo ultrassonográfico morfológico com história de mal- formação cardíaca com arritmia, diagnosticada em exame ultrassonográfico de rotina. Em nossa avaliação, os achados ecográficos revelaram a presença de massa intracardíaca volumosa ocupando o átrio direito e abaulando o septo interatrial. A massa tinha aspecto hiperecogênico e homogêneo e media $25 \times 21 \mathrm{~mm}$ nos maiores diâmetros (Figura 1). O feto apresentava quadro de taquiarritmia acentuada persistente (média de 263 batimentos cardíacos/minuto), com sinais claros de insuficiência cardíaca congestiva à avaliação ecográfica bidimensional e ao modo Doppler, e hidropisia (Figuras 2, 3 e 4).

Figura 1. Imagem cardíaca fetal no plano das quatro câmaras. Evidencia-se volumosa massa ecogênica ocupando o átrio direito, abaulando o septo interatrial (setas). $A D$, átrio direito; VD, ventrículo direito; VE, ventrículo esquerdo.

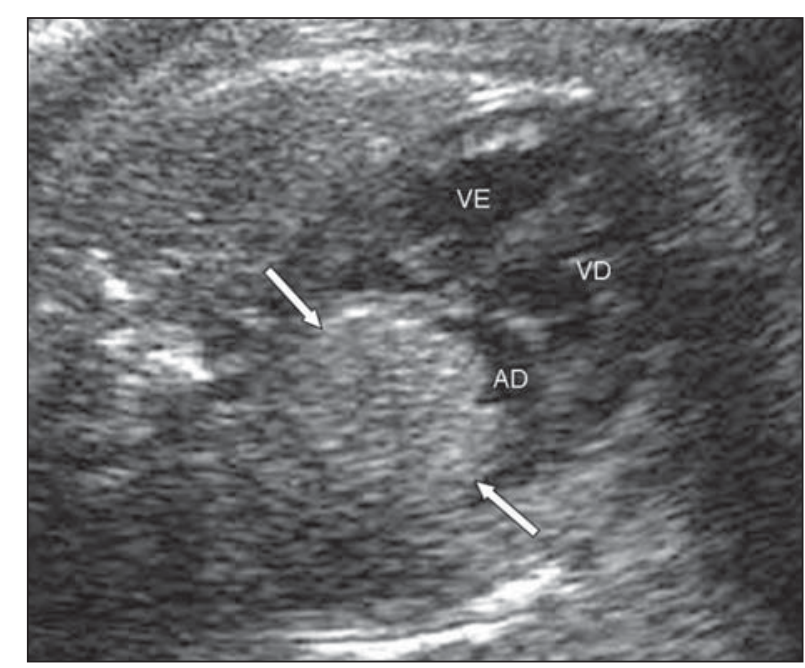




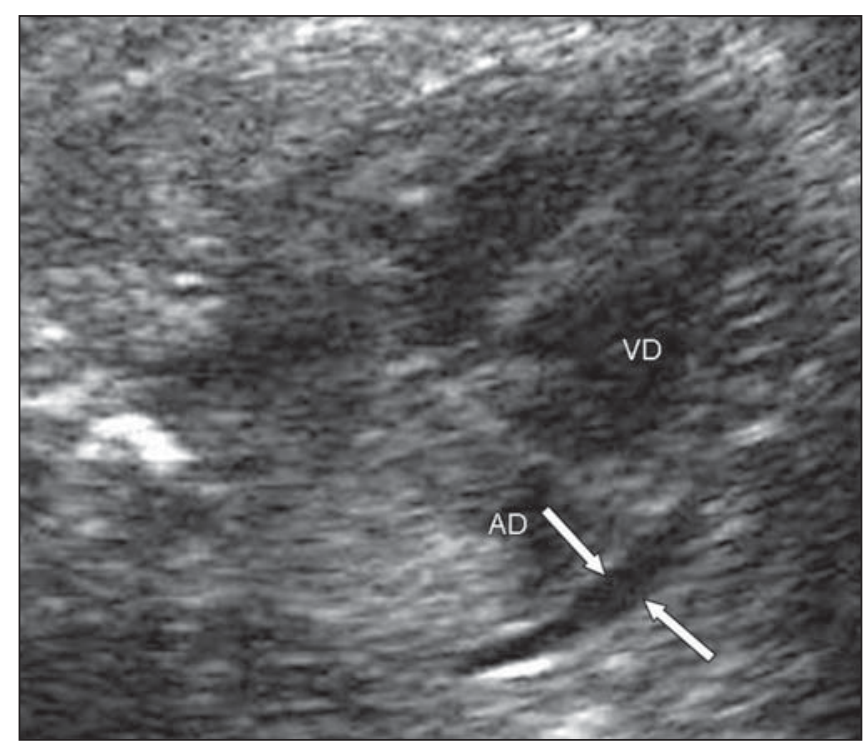

Figura 2

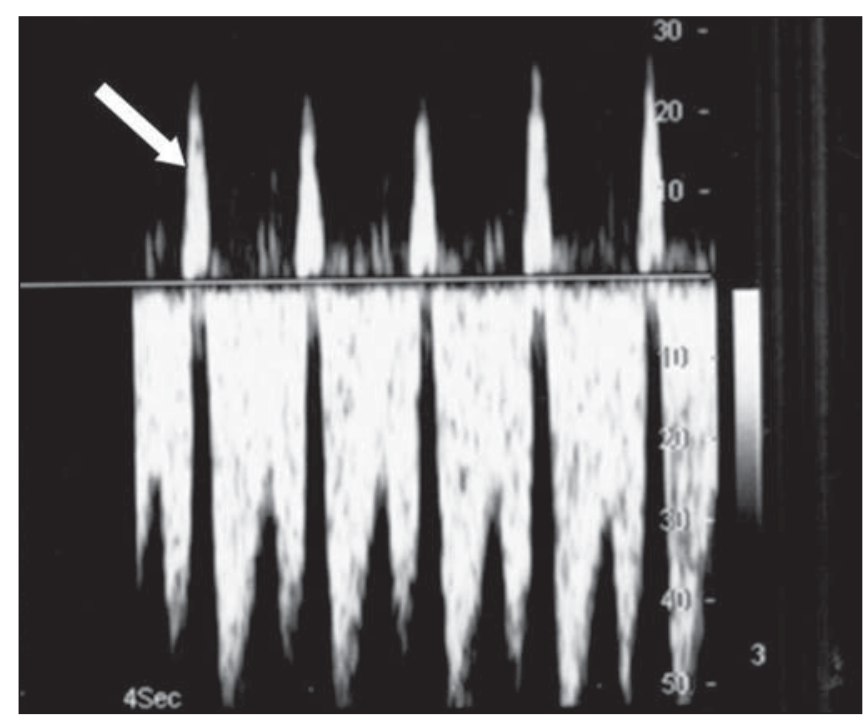

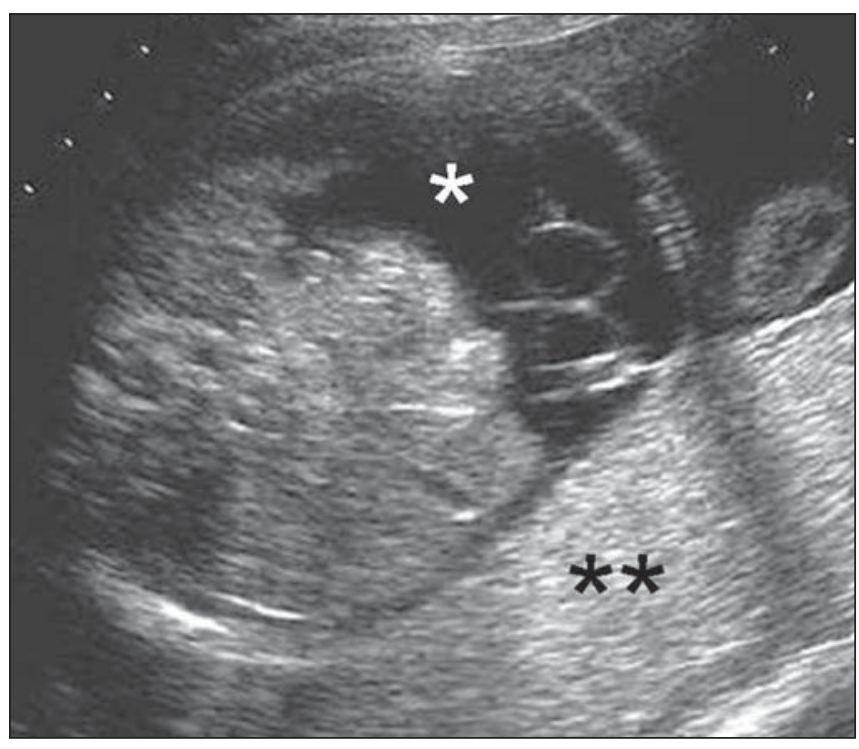

Figura 3

Figura 2. Insuficiência cardíaca. Imagem evidenciando derrame pericárdico (setas). $A D$, átrio direito; $V D$, ventrículo direito.

Figura 3. Hidropisia fetal. Imagem do abdome fetal evidenciando ascite volumosa $\left({ }^{*}\right)$. Observa-se ainda placentomegalia $(* *)$.

Figura 4. Insuficiência cardíaca. Ducto venoso evidenciando onda "A" reversa durante a contração atrial (seta).

Figura 4

A despeito da terapêutica instituída, o feto evoluiu para óbito em dez dias. A necropsia confirmou a suspeita diagnóstica ultrassonográfica pré-natal de rabdomioma cardíaco.

\section{DISCUSSÃO}

Os tumores cardíacos, embora representando condição rara, têm sua incidência aumentada para até $0,2 \%$ em centros de referência para cardiopatia pediátrica ${ }^{(6)}$. Eles podem apresentar repercussões hemodinâmicas variadas. De acordo com sua localização, tamanho e número, podem levar a obstrução do fluxo sanguíneo e alteração da fração de ejeção ventricular, causando arritmia, insuficiência cardíaca congestiva, efusão pericárdica, cardiomegalia, hidropisia e óbito fetal ${ }^{(5,7)}$.

Quanto aos tipos histológicos, o rabdomioma cardíaco ou hamartoma representa a linhagem histológica mais comum no período pré-natal ${ }^{(6,7)}$. A frequente associação desta entidade com a esclerose tuberosa, doença de transmissão autossômica dominante, enfatiza a importância do seu rastreamento diagnóstico no feto e em seus genitores, pois a esclerose tuberosa chega a acometer mais de $50 \%$ dos fetos com tumores cardíacos primários ${ }^{(7)}$. No presente caso, a avaliação ultrassonográfica do sistema nervoso central do feto não detectou alterações suspeitas de comprometimento intracraniano, e os genitores estão sendo avaliados para esclerose tuberosa. Os fibromas, hemangiomas e teratomas acometem o coração fetal mais raramente ${ }^{(\mathbf{8 , 9})}$. Os fibromas são usualmente únicos e envolvem comumente a parede do ventrículo esquerdo ou o septo interventricular ${ }^{(\boldsymbol{( 6 )}}$. Os teratomas têm origem no pericárdio, apresentando-se únicos, heterogêneos, encapsulados e de grandes dimensões, atingindo frequentemente a base do coração à direita ${ }^{(8)}$. Já os hemangiomas cardíacos são raros na vida intrauterina e na infância ${ }^{(7)}$. Embora os mixomas cardíacos sejam prevalentes na idade adulta, há na literatura apenas um relato deste tipo histológico de tumor diagnosticado na vida intrauterina ${ }^{(\mathbf{1 0})}$. 
À ultrassonografia, o rabdomioma cardíaco usualmente revela-se a partir do final do segundo trimestre como massa sólida bem delimitada, homogênea, hiper-refringente, única ou múltipla, de dimensões variadas $^{(2,6,7)}$. Frequentemente, acometem o ventrículo esquerdo $(60 \%)$ e em menor proporção, o ventrículo direito $(26 \%)^{(6)}$. Disritmias cardíacas são observadas em $16 \%$ a $47 \%$ dos $\operatorname{casos}^{(5)}$. Diante de comprometimento da função cardíaca, terapia com digitálicos ou outros antiarrítmicos, por via oral materna ou por via intravenosa fetal (cordocentese), e o parto pré-termo terapêutico devem ser considerados ${ }^{(6,7)}$. A excisão cirúrgica intrauterina desses tumores foi sugerida para tratar fetos imaturos com hidropisia, mas ainda não existe consenso quanto à indicação desta terapêutica ${ }^{(11)}$.

Todavia, na maioria das vezes os rabdomiomas cardíacos são tipicamente bem tolerados, com risco de óbito fetal estimado em $4 \%$ a $6 \%$ dos casos ${ }^{(7)}$. Há relatos de regressão espontânea do tumor, particularmente no terceiro trimestre ${ }^{(7)}$. Em estudo retrospectivo, avaliaram-se 19 casos de tumores cardíacos fetais, sendo observada regressão parcial ou completa dessas mas- sas em 8 pacientes, sendo que 10 desenvolveram, posteriormente, quadro clínico de esclerose tuberosa ${ }^{(7)}$. De fato, uma evolução perinatal benigna, com progressiva diminuição do tumor e completa resolução, pode ser observada em mais de $80 \%$ dos casos durante a infância ${ }^{(6,7)}$.

\section{CONCLUSÃO}

O diagnóstico pré-natal dos tumores cardíacos fetais é passível de realização por meio de exame ecográfico minucioso, com raros casos de diagnóstico falso-positivo ${ }^{(\mathbf{1 2})}$. O reconhecimento precoce desta afecção possibilita seguimento pré-natal especializado e, quando indicada, terapêutica apropriada, contribuindo, dessa forma, para a redução da morbimortalidade perinatal.

\section{REFERÊNCIAS}

1. D'Ippolito G, Medeiros RB. Exames radiológicos na gestação. Radiol Bras. 2005;38:447-50.

2. McAllister HA Jr. Primary tumors of the heart and pericardium. Patol Annu. 1979; 14 Pt 2:325-55.

3. Zhou QC, Fan P, Peng QH, et al. Prenatal echocardiographic differential diagnosis of fetal cardiac tumors. Ultrasound Obstet Gynecol. 2004;23: 165-71.

4. Araujo Júnior E, Zanforlin Filho SM, Pires CR, et al. Spatio-temporal image correlation (STIC): nova técnica para avaliação do coração fetal. Radiol Bras. 2006;39:373-7.

5. Kagan KO, Schmidt M, Kuhn U, et al. Ventricular outflow obstruction, valve aplasia, bradyarrhythmia, pulmonary hypoplasia and non-immune fetal hydrops because of a large rhabdomyoma in a case of unknown tuberous sclerosis: a prenatal diagnosed cardiac rhabdomyoma with multiple symptoms. BJOG. 2004;111:1478-80.

6. Holley DG, Martin GR, Brenner JI, et al. Diagnosis and management of fetal cardiac tumors: a multicenter experience and review of published reports. J Am Coll Cardiol. 1995;26:516-20.

7. Bader RS, Chitayat D, Kelly E, et al. Fetal rhabdomyoma: prenatal diagnosis, clinical outcome, and incidence of associated tuberous sclerosis complex. J Pediatr. 2003;143:620-4.

8. Riskin-Mashiah S, Moise KJ Jr, Wilkins I, et al. In utero diagnosis of intrapericardial teratoma: a case for in utero open fetal surgery. Prenat Diagn. 1998;18:1328-30.

9. Tongsong T, Sirichotiyakul S, Sittiwangkul R, et al. Prenatal sonographic diagnosis of cardiac hemangioma with postnatal spontaneous regression. Ultrasound Obstet Gynecol. 2004;24:207-8.

10. Paladini D, Tartaglione A, Vassallo M, et al. Prenatal ultrasonographic findings of a cardiac myxoma. Obstet Gynecol. 2003;102(5 Pt 2):1174-6.

11. Parry AJ, Carroll S, Lotto A, et al. Median sternotomy as an exit procedure in a child with massive pericardial tumor. J Pediatr Surg. 2004;39: 1408-10.

12. Tehrani M, Vettraino IM, Chang CH. Localized nodular hypertrophy mimicking rhabdomyoma in the fetal heart: prenatal sonographic and pathology findings. Pediatr Dev Pathol. 2004;7:192-7. 